\title{
Evaluation of Primary Brain Tumor with Magnetic Resonance Imaging
}

\author{
S. Vidyasagar ${ }^{1}$, Abubacker Sulaiman $\mathrm{F}^{2}$ \\ ${ }^{1}$ Associate Professor, ${ }^{2}$ Professor, Dhanalakshmi Srinivasan Medical College and Hospital, Siruvachur, Perambalur, India
}

Corresponding author: Dr. Vidyasagar, No:32, Rajresidency, Ramarayar Agraharam, Tennur, Trichy Pin-620017, India

DOI: http://dx.doi.org/10.21276/ijcmsr.2019.4.1.32

How to cite this article: S. Vidyasagar, Abubacker Sulaiman F. Evaluation of primary brain tumor with magnetic resonance imaging. International Journal of Contemporary Medicine Surgery and Radiology. 2019;4(1):A124A128.

\section{A B S T R A C T}

Introduction: With the emergence of advanced MRI techniques, the use of conventional MR imaging has been reported to be relatively low. It is the most widely used non-invasive technique for brain tumor grading. Advanced MR techniques, such as perfusion-weighted imaging (PWI), diffusion-weighted imaging (DWI), and magnetic resonance spectroscopy (MRS), could predict neoplastic histology, but their added value over conventional MRI is still controversial. Hence, the aim of the present study was to assess the primary brain tumour with the help of magnetic resonance imaging among patients attending the Chettinad Hospital and Research Institute, Kelambakkam.

Material and methods: The present study was a prospective study which was conducted among 30 patients with suspected brain tumor which were selected for undergoing MRI brain with IV contrast in Chettinad Hospital and Research Institute, Kelambakkam. All these patients were imaged on GE sigma 1.5 Tesla scanner, with the gradient strength $33 \mathrm{mT} / \mathrm{m}$ at aslew rate of $120 \mathrm{mT} / \mathrm{m} / \mathrm{ms}$, an 8 channel head coil is used for signal receiver.

Results: In this study, 30 patients were examined with the symptoms of brain tumor. Out of which, 23 patients were diagnosed with primary brain tumor. Many MRI sequences were done to rule out brain tumor. T2, T2 FLAIR, T1 FAT saturation pre contrast and post contrast were found to be best sequences to rule out primary brain tumor.

Conclusion: MR is ideal imaging technique for evaluation of intracranial lesions because of excellent anatomic details as well as delineate pathological lesions discreetly. The diagnostic accuracy of MRI in the evaluation of brain tumors and tumors like lesions increases when combined with DWI and MRS with conventional MR imaging.

Keywords: Magnetic Resonance Imaging, Brain Tumor, Gliomas, Computed Tomography

\section{INTRODUCTION}

Brain tumor referred to the aggregation of abnormal cells in some tissues of the brain. According to the brain tumors origin, they are divided into two categories, primary and metastatic brain tumors. The origin of primary brain tumors is in the brain, while metastatic brain tumors originate from other body parts. Tumors can be cancerous (or malignant) or non-cancerous (or benign). Malignant brain tumors grow fast and spread to other areas of the brain and spine and compared to benign tumors, they are more life-threatening. Gliomas are considered as the most common type of primary brain tumor in adults. ${ }^{1}$

According to the World Health Organization grading system, gliomas are diagnosed in grades of severity from I to IV. Grade I tumors have cells that are benign and are approximately normal in appearance. Grade II tumors have cells that appear to be slightly abnormal. Grade III tumors have cells that are malignant and clearly abnormal. The most severe type of brain tumors that contain fast-spreading and abnormal cells are considered as Grade IV. Glioblastoma multiforme (GBM) are quintessential tumors of this type. Meningioma tumors arise from a layer of tissue called the meninges. ${ }^{2}$

Meninges cover the brain and spinal cord and act as protector. They are mostly considered as benign tumors, because they grow at a slow pace and are also less likely to spread. Pituitary tumors develop in the pituitary gland and account for $14 \%$ of all primary intracerebral tumors, with most of them are due to spontaneous mutation and some are due to inherited genetic defects. These tumors are also benign and they are much less likely to spread. Although these tumors are considered benign, they can cause serious health problems due to their presence in sensitive areas of the brain. ${ }^{3}$

Early detection plays a major role in treatment and recovery of the patients. Diagnosing a brain tumor and its grade usually undergoes a complicated and time-consuming process. Usually, the patient refers to MRI when the brain tumor has grown sufficiently and several harassing symptoms have appeared. After examining the brain images, if tumor existence is suspected, the patient's brain biopsy comes to action. Unlike MR, biopsy has an invasive procedure and in some cases, it may even take up to a month for a definite answer. MRI specialists perform techniques such as perfusion to grade tumor and biopsy to confirm. It should be noted that in recent years some novel methods have been introduced in 
order to grade brain tumors other than biopsy. ${ }^{4,5}$

Evaluation of the brain by conventional MRI involves largely qualitative analysis of observed pathology. The use of intravenous gadolinium-based contrast agents (GBCA's) is useful for detecting regions of blood brain barrier (BBB) disruption, which enhance on T1 weighted images following the administration of contrast agent. This modality has emerged as a crucial component in the diagnosis and management of brain tumors, largely due to the fact that it provides superior resolution and anatomic detail compared with its CT counterpart. ${ }^{6}$

The most clinically relevant information that can be obtained from conventional MRI with regard to cranial spaceoccupying lesions includes morphological data such as lesion number, size, location, and extension. The standard technique for the brain tumor diagnosis is MRI which is a non-invasive method that offers good soft tissue contrast. It is utilized in combination with other imaging methods, such as computed tomography $(\mathrm{CT})$, positron emission tomography (PET) and magnetic resonance spectroscopy (MRS) to supply the most precise info about tumor morphology and metabolic process. MRI remains the accepted standard and for that reason we will focus on MRI based methods. ${ }^{7,8}$

The evolution of MRI technology has made possible the emergence of more advanced MRI techniques beyond conventional anatomic MRI. While conventional MRI lacks biologic specificity and is unable to provide physiologic detail of tumor biology, these techniques show promise in providing a more complete evaluation of tumor biology and clinical status. ${ }^{9}$ Hence, the aim of the present study was to assess the primary brain tumour with magnetic resonance imaging among patients attending the Chettinad Hospital and Research Institute, Kelambakkam.

\section{MATERIAL AND METHODS}

The present study was a prospective study which was conducted among 30 patients with suspected brain tumor which were selected for undergoing MRI brain with IV contrast in Chettinad Hospital and Research Institute, Kelambakkam. All these patients were imaged on GE sigma 1.5 Tesla scanner, with the gradient strength $33 \mathrm{mT} / \mathrm{m}$ at aslew rate of $120 \mathrm{mT} / \mathrm{m} / \mathrm{ms}$, an 8 channel head coil is used for signal receiver. A variety of patients with different age group were examined. For selection of patients, simple random sampling technique was used for this study. Patients with symptoms of brain tumor that includes chronic headache, vomiting, seizure and vision disorder including vertigo were included in the present study. Patients with metallic implants, claustrophobic patients, with cardiac pacemaker and pregnant patients were excluded from the study. The gadolinium MR contrast was injected at a dose of $0.1 \mathrm{mmol} /$ $\mathrm{kg}$ of body weight intravenously. About 23 patients showed various types of primary brain tumor among which 7 of them showed finding of metastases.

\section{Examination Technique and MRI Sequences}

Patients were subjected to routine brain sequences like DWI (diffusion weighted images), axial T2 flair, axial T2, axial T1 flair, axial T2*, coronal T2 flair, sagittal T1 flair, susceptibility weighted images (SWI), axial T1 fat saturation (pre contrast), axial T1 fat saturation (post contrast), coronal $\mathrm{t} 1$ fat saturation (post contrast) and sagittal T1 fat saturation post contrast.

\section{Image planning}

Sequences are planned based on the initial localized images obtained in three plane localizer.

\section{STATISTICAL ANALYSIS}

The data was analyzed with the help of statistical procedures with SPSS version 21.0. Descriptive statistic was presented in the form of tables and graphs whereas a multivariate logistic regression model was applied to assess the combined and independent values of predictor variables with the help of inferential statistics.

\section{RESULTS}

In this study, 30 patients were examined with the symptoms

\begin{tabular}{|c|c|c|c|c|c|c|c|c|}
\hline Parameter & $\begin{array}{c}\text { T2 } \\
\text { Flair }\end{array}$ & T2 TSE & T1 SE & $\begin{array}{c}\text { T2 } \\
\text { FLAIR }\end{array}$ & T1 SE & $\begin{array}{c}\text { T1 } \\
\text { SE (pre } \\
\text { contrast) }\end{array}$ & $\begin{array}{c}\text { T1 } \\
\text { SE (post } \\
\text { contrast) }\end{array}$ & $\begin{array}{c}\text { T1 } \\
\text { SE (post } \\
\text { contrast) }\end{array}$ \\
\hline Mode of acquisition & $2 \mathrm{D}$ & $2 \mathrm{D}$ & $2 \mathrm{D}$ & $2 \mathrm{D}$ & $2 D$ & $2 \mathrm{D}$ & $2 \mathrm{D}$ & $2 \mathrm{D}$ \\
\hline Plane & Axial & Axial & Axial & Coronal & Saggital & Axial & Axial & Coronal \\
\hline FOV $(\mathrm{mm})$ & 24 & 24 & 24 & 28 & 26 & 24 & 24 & 28 \\
\hline TR & 6500 & 4000 & 800 & 6500 & 825 & 830 & 830 & 830 \\
\hline TE & 800 & 650 & 75 & 800 & 80 & 90 & 90 & 90 \\
\hline \multirow[t]{2}{*}{ matrix } & $288 * 22$ & $322 * 256$ & $256 * 19$ & $256 * 192$ & $256 * 19$ & $326 * 25$ & $326 * 25$ & $326 * 256$ \\
\hline & 6 & & 6 & & 2 & 6 & 6 & \\
\hline Fat sat & No & No & No & No & No & Yes & Yes & YES \\
\hline Slice & 4 & 4 & 4 & 4 & 4 & 4 & 4 & 4 \\
\hline \multicolumn{9}{|l|}{ Thickness (mm) } \\
\hline Inter slice (mm) & 1 & 1 & 1 & 1 & 1 & 1 & 1 & 1 \\
\hline NEX & 2 & 2 & 2 & 1 & 2 & 2 & 2 & 2 \\
\hline Timing (min) & 3.28 & 1.45 & 1.05 & 1.48 & 1.15 & 3.45 & 3.45 & 3.55 \\
\hline IV contrast & No & No & No & No & No & No & Yes & Yes \\
\hline
\end{tabular}



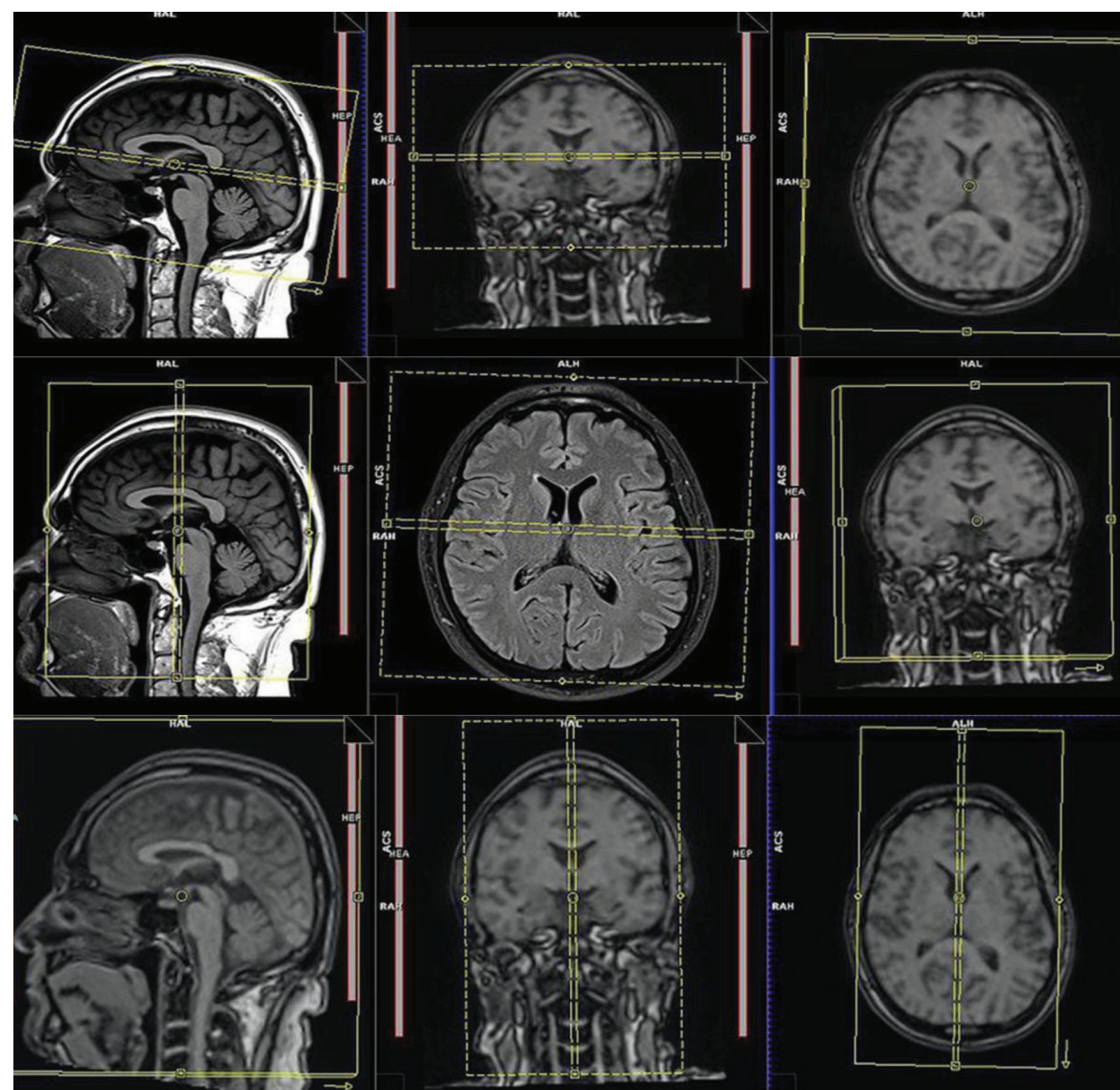

Figure-1: Pre and post contrast axial, coronal and sagittal views among the study subjects
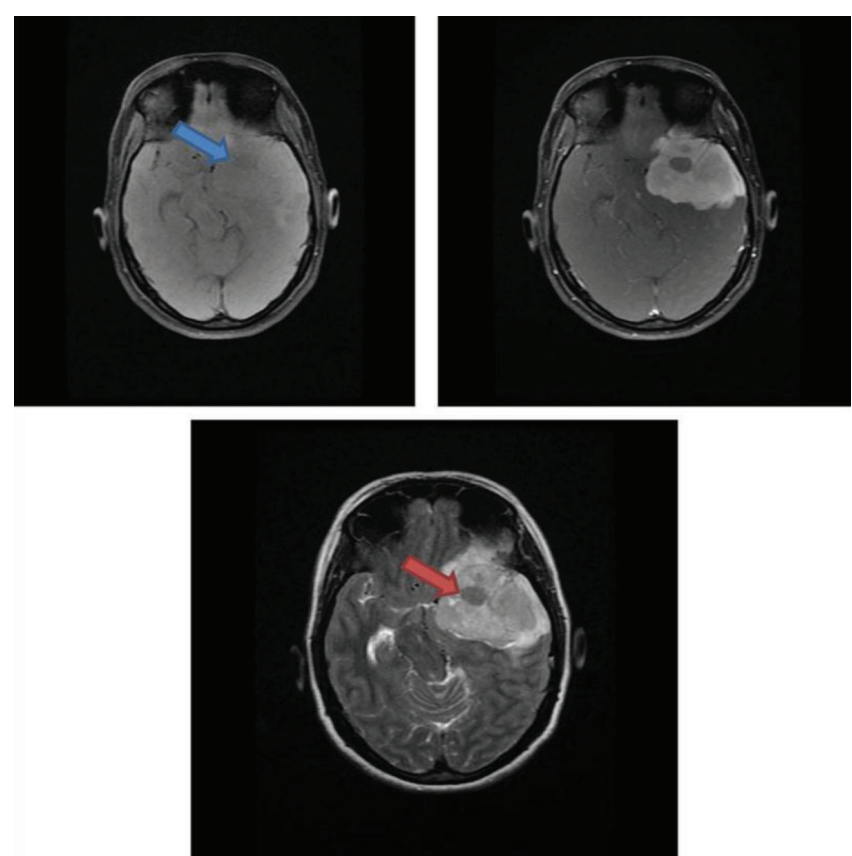

Figure-2: Solid mass, isointense grey matter in T1 (blue arrow)/T2W sequences. The lesion shows mostly solid enhancement with non-enhancing T2 (red arrow) hypointense area. Features consistent with sphenoid wing meningioma
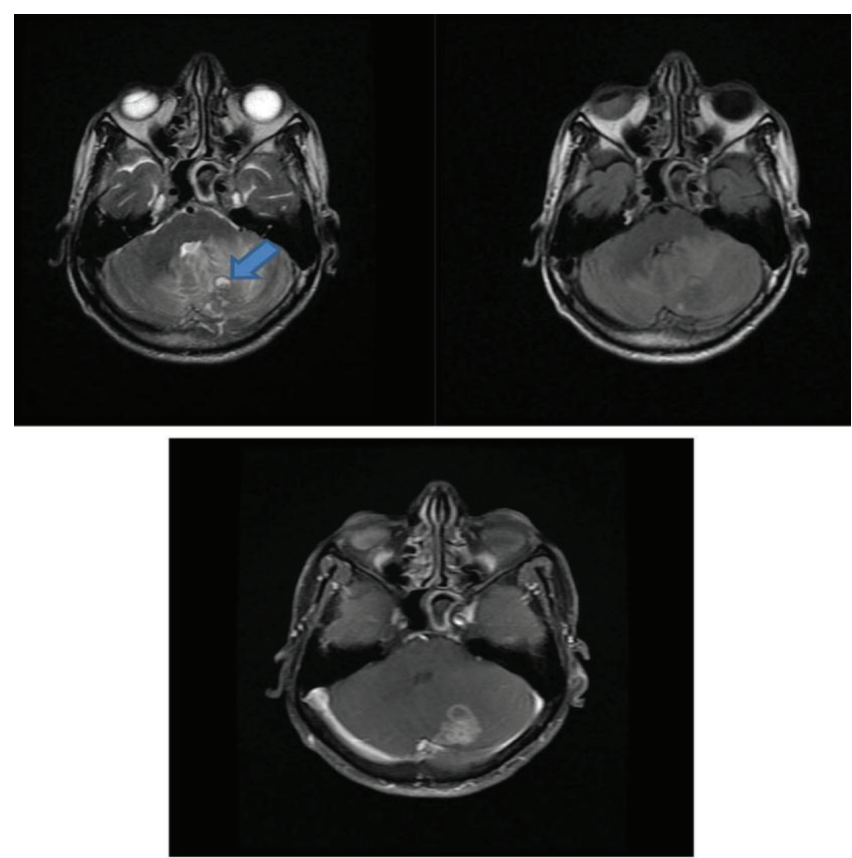

Figure-3: Enhanced lobulated prominently T2 (blue arrow) intermediate intense mass with few tiny cystic areas within is noted involving grey-white matter of paramedian aspect of left cerebellar hemisphere. Possibilities include (in the order of probability) metastasis, atypical hemangioblastoma, astrocytoma, medulloblastoma. 
of brain tumor. Out of which, 23 patients were diagnosed with primary brain tumor. Many MRI sequences were done

\section{$\mathrm{T} 1$}

T2

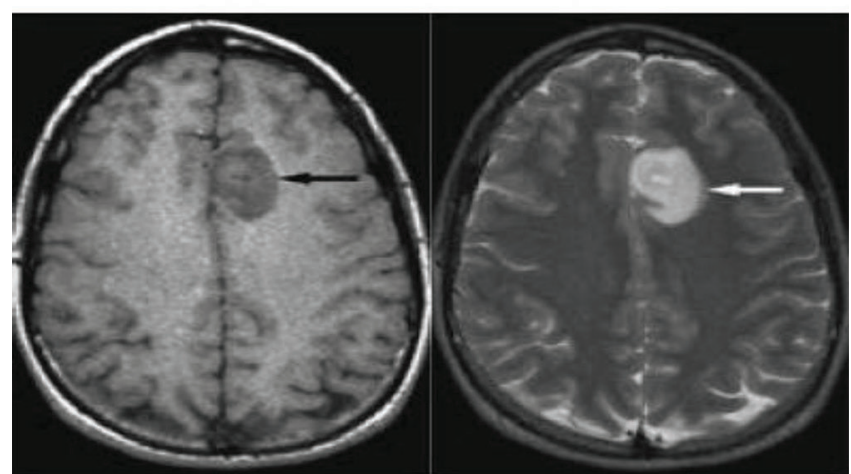

Figure-4: The tumor appears hypointense in t1 weighted image and hyper intense in $t 2$ weighted image

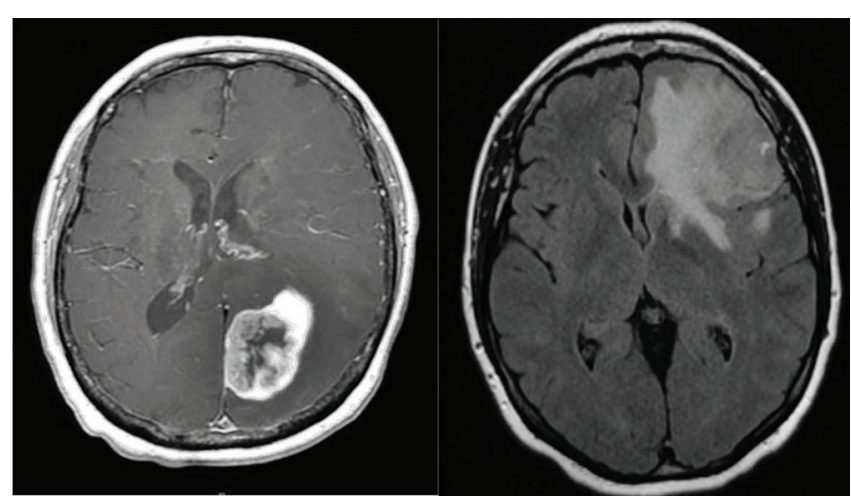

Figure-5: Tumor site enhanced using MRI contrast gadolinium

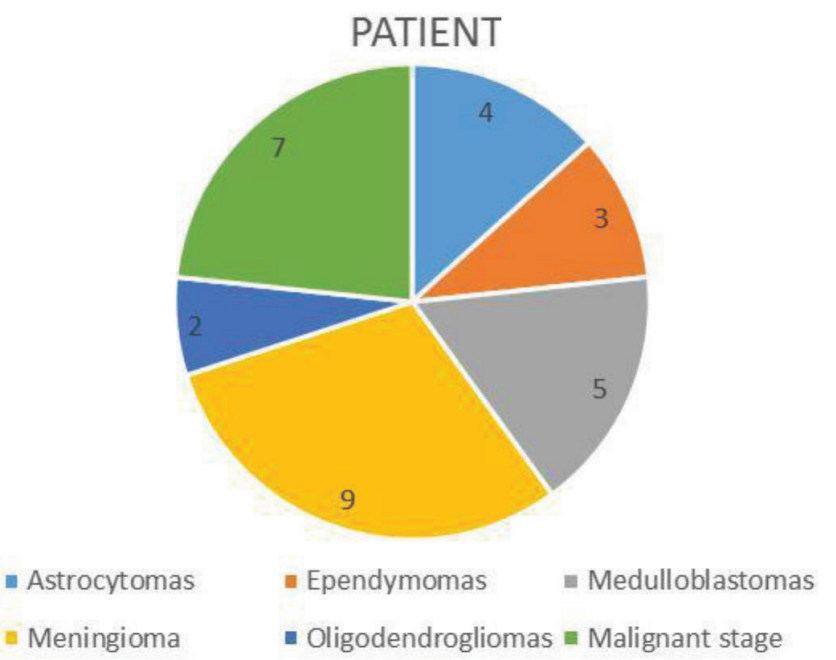

Graph-1: Various types of primary brain tumours among the study subjects

\begin{tabular}{|l|c|c|c|}
\hline Tissue & T1 & T2 & Flair \\
\hline CSF & Dark & Bright & Dark \\
\hline White Matter & Light & Light Gray & Dark Gray \\
\hline Cortex & Grey & Dark Gray & Light Gray \\
\hline Abnormality & Dark & Bright & Bright \\
\hline \multicolumn{4}{|r|}{ Table-2: Appearance of brain in MRI sequences } \\
\hline
\end{tabular}

to rule out brain tumor. T2, T2 FLAIR, T1 FAT saturation pre contrast and post contrast were found to be best sequences to rule out primary brain tumor (Figure no. 1, 2 and 3).

It was found that in few cases, that there were some abnormalities which were not clearly visible, but were visualized in a better detail with contrast administration. In addition, there were special sequences used for better visualization and evaluation. In some case, there was restriction in the Diffusion Weighted Imaging (DWI) which helped in ruling out the tumor. In some cases, MR Spectroscopy allowed for characterization of tumor by varying levels of choline and NAA. Thus, MRI enabled evaluation in grades of tumor (Figure no. 4 and 5). Graph no. 1 shows the various types of tumors among the study subjects out of which 7 patients were in malignant stage.

\section{DISCUSSION}

Imaging plays a pivotal role in the clinical management of human brain tumors. Diagnostic anatomical features such as the presence (or absence) of contrast enhancement, hemorrhage, calcification and/or macroscopic necrosis are readily assessed through standard magnetic resonance imaging (MRI) and computed tomography (CT). Secondary pathologic conditions such as mass effect, edema and herniation are also assessed via CT and MRI. Serial imaging with gadolinium-enhanced MRI is routinely employed to evaluate the success or failure of therapy and for post-therapy surveillance in order to detect early tumor recurrence. ${ }^{10}$

To investigate the biological nature of tumors rather than the subsequent anatomical changes advances in MRI are proven to be helpful for researchers. Imaging techniques such as MR diffusion, functional, perfusion, and spectroscopic imaging provides opportunities to improve diagnostic, prognostic, and therapeutic assessments. Recently, MRI is being applied to grade gliomas, guide stereotactic biopsies, to see the difference between non-glial and extra-axial tumors. Additionally, it also helps to find out the difference between primary and metastatic tumors, monitor treatment response, distinguish between recurrent tumors and treatment necrosis, and eliminate neoplastic-mimicking lesions, such as stroke and tumefactive demyelinating lesions. ${ }^{11}$

It was found from the literature that gliomas showed increased Cho and decreased NAA, and increasing Cho/ $\mathrm{Cr}$ and $\mathrm{Cho/Cho} \mathrm{(normal)} \mathrm{ratios} \mathrm{with} \mathrm{increasing} \mathrm{glioma}$ grades. This increment in Cho is attributable to accelerated cell membrane synthesis and turnover; however, Cho is underestimated and may be unreliable in heterogeneous tumors due to internal hemorrhage, necrosis, or cysts. ${ }^{12}$

In the assessment of primary brain tumors, conventional MRI is most commonly used technique. However, when grading brain tumors, higher accuracy is very much needed. Advanced MR techniques helps in gaining additional information related to histological features of the tumor, as grade of neovascularization, cellularity, and mitotic index. The validity of conventional and advanced MR in grading tumors has been widely reported in the medical literature. ${ }^{13}$ It has been found from literature that very few attempts have been made to combine different MRI techniques in grading tumors and the study done by Yoon et al. has revealed that 
combined conventional MRI with PWI, DWI, and MRS in a group of patients diagnosed with cerebral gliomas. It was found from the study that there were no significant differences in the diagnostic performances of any of those MR imaging techniques. Furthermore, a study conducted by Caulo et al reported that the information provided by these advanced MR techniques in the assessment of tumor grade in gliomas but, in their analysis, ADC maps were only used to define different tumoral regions in order to guide ROIs' placement. Thus, the ADC values were not calculated to differentiate grade of aggressiveness. In the current prospective study, it was analyzed that a series of variables for conventional MRI and advanced techniques (PWI, DWI, and MRS) to determine whether a combination of techniques was better than conventional MRI alone and, unlike in Caulo et al $\mathrm{ADC}$ values were used to assess tumor grade. ${ }^{14,15}$

It was seen from the previous studies that analyzed differences between tumor grades were limited to gliomas. However, in the present study, there was inclusion of all the primary brain tumors in order to mimic conditions of clinical practice, where radiologist has to provide a presumptive diagnosis of malignancy before surgery, regardless of the histological type. It was found that some tumors were non-gliomas because of the reduced frequency of these types of tumors.

Early detection plays an important role in recovery and treatment of the patients. Diagnosing a brain tumor and its grade usually undergoes a complicated and time-consuming process. Hence, the patient should be referred to MRI when the brain tumor has grown sufficiently. ${ }^{16}$

\section{CONCLUSION}

MRI is a best instrument for evaluating patients with primary brain tumors. Early detection of primary brain tumor helps in treatment because of treatment-associated imaging changes. Other modalities under MRI that includes spectroscopy, diffusion and perfusion helps to evaluate the nature of the tumor.

\section{REFERENCES}

1. Black PM. Brain tumors. N Engl J Med 1991; 324(22):1555-64.

2. Kelly PJ. Gliomas: survival, origin and early detection. Surg Neurol Int 2010;1.

3. Litjens G, Kooi T, Bejnordi BE, Setio AAA, Ciompi F, Ghafoorian M, et al. A survey on deep learning in medical image analysis. Med Image Anal 2017.

4. Lo C-S, Wang C-M. Support vector machine for breast MR image classification. Comput Math Appl 2012;64(5):1153-62.

5. Trigui R, Mitéran J, Walker PM, Sellami L, Hamida AB. Automatic classification and localization of prostate cancer using multi-parametric MRI/MRS. Biomed Signal Process Control 2017;31(2):189-98.

6. Louis DN, Perry A, Reifenberger G, Von Deimling A, Figarella- Branger D, Cavenee WK, et al. The 2016 World Health Organization classification of tumors of the central nervous system: a summary. Acta Neuropathol 2016; 131(6):803-20.

7. Clark K, Vendt B, Smith K, Freymann J, Kirby J, Koppel P, et al. The Cancer Imaging Archive (TCIA): maintaining and operating a public information repository. J Digit Imaging 2013; 26(6):1045-57.

8. Zhang Y, Dong Z, Wu L, Wang S. A hybrid method for MRI brain image classification. Expert Syst Appl 2011; 38 (8):10049-53.

9. Saritha M, Joseph KP, Mathew AT. Classification of MRI brain images using combined wavelet entropy based spider web plots and probabilistic neural network. Pattern Recogn Lett 2013; 34(16):2151-6.

10. Dean BL, Drayer BP, Bird CR, Flom RA, Hodak JA, Coons SW, Carey RG: Gliomas: classification with MR imaging. Radiology 1990; 174(3):411-415. 32.

11. Watanabe M, Tanaka R, Takeda N: Magnetic resonance imaging and histopathology of cerebral gliomas. Neuroradiology 1992; 34:463-469.

12. Majos C, Alonso J, Aguilera C, Serrallonga M, PerezMartin J, Acebes JJ, Arus C, Gili J: Proton magnetic resonance spectroscopy ((1)H MRS) of human brain tumours: assessment of differences between tumour types and its applicability in brain tumour categorization. Eur Radiol 2003; 13:582-591. 26.

13. Hakyemez B, Erdogan C, Ercan I, Ergin N, Uysal S, Atahan S: High-grade and low-grade gliomas: differentiation by using perfusion MR imaging. Clin Radiol 2005; 60:493-502

14. Yoon JH, Kim J, Kang WJ, Sohn C-H, Choi SH, Yun TJ, Eun Y, Song YS, Chang K-H: Grading of cerebral glioma with multiparametric MR imaging and 18F-FDG-PET: concordance and accuracy. Eur Radiol 2014; 24:380-389.

15. Caulo M, Panara V, Tortora D, Mattei PA, Briganti C, Pravatà E, Salice S, Cotroneo AR, Tartaro A: Datadriven grading of brain gliomas: a multiparametric MR imaging study. Radiology 2014; 272:494-503.

16. Fayed N, Modrego PJ: The contribution of magnetic resonance spectroscopy and echoplanar perfusionweighted MRI in the initial assessment of brain tumours. J Neurooncol 2005; 72:261-265.

Source of Support: Nil; Conflict of Interest: None

Submitted: 10-01-2018; Accepted: 17-02-2018; Published online: 30-03-2019 